\title{
Optimization of 3G Radio Network Planning Using Tabu Search
}

\author{
Yufei Wu and Samuel Pierre
}

\begin{abstract}
There are many factors that have to be considered during planning phase of mobile radio networks. Some of the most important factors are: quality of service (QoS), cost of implementation and provisioning, traffic coverage ratio, and resource utilization. This paper analyzes economic aspects of $3 G$ mobile radio network deployment and presents a planning model which explicitly takes into account some important factors such as QoS and investment cost. It emphasizes the impact of planning objectives and network element cost structures on 3G radio network deployment. The planning problem can be NP-hard, which is approximately tackled by a metaheuristic optimization method - tabu search algorithm. Numerical experiments with realistic problem sizes are conducted to describe some important aspects of efficient $3 G$ network planning and demonstrate the efficiency and the practicality of the tabu search algorithm.
\end{abstract}

Index Terms-3G, Mobile network planning, 3G, Tabu Search, Optimization.

\section{INTRODUCTION}

$\mathrm{E}$ VERY cellular network deployment requires planning and

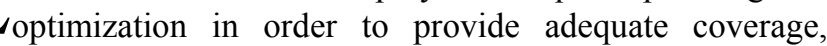
capacity, and quality of service (QoS). Mobile radio network planning and optimization is a very complex task, as many aspects must be taken into account, including the topology, morphology, traffic distribution, existing infrastructure. Depending on how these and other factors are incorporated into the planning process, there can be many possible strategies. During planning process, each of these factors can be either part of the objective function or subject to certain constraints or neglected. As shown in [1][3][13], Mobile network planning problem can be NP-hard.

A good planning objective is extremely important in selecting an effective deployment scenario. In [2][3][4], the models proposed are for both microcells and picocells in coverage limited environments. These approaches use an objective function directly related to path loss which is optimized to determine base station locations. In [5], authors propose a model which considers interference limited environments and use an objective function which directly

Y. F. Wu is with the Research Center for Information and Communication Technology , University of Trinidad and Tobago, Trinidad and Tobago. (phone: 1 868-642 8888; fax: 1 868-643-5615; e-mail: yufei.wu@ utt.edu.tt).

S. Pierre is with the Computer Engineering Department, Ecole Polytechnique de Montreal, Quebec, Canada, (e-mail: samuel. pierre@polymtl.ca). related to QoS. In [6], the goal of mobile network planning and optimization is to cover the maximum number of subscribers in an effective manner. In [7] a profit maximization model is presented. In [8] a cost minimizing planning and optimization model is developed. Most of those previous studies have been carried out for $2 \mathrm{G}$ mobile networks, and have not revealed enough information about efficient mobile network deployment configurations. Planning $3 \mathrm{G}$ mobile networks, such as UMTS, is even more sensitive to the planning objective due to the inherent CDMA features [9]. In [10], a net revenue maximizing model is presented, which takes as given a set of base station (BS) locations with corresponding costs, the amount of available bandwidth, the maximum demand for service in each geographical area, and the revenue potential in each customer area. In [11], a net revenue maximizing model for WCDMA networks has been investigated, which can help a network planner with the selection of BS sites and the calculation of service capacity. In [12], the cost efficient planning of a single frequency network (SFN) is proposed, which is compared with coverage efficient planning scenario and coverage planning with saving best cost configuration scenario. In [22], the author proposed an approach which is based on the application of the powerful optimization technique known as Particle Swarm Optimization (PSO). First, the working area is represented by a suitable model showing topological and morphological information and the places for the candidate sites. After that, a solution representat on is proposed converting each network plan to a bit stream. Finally, the PSO algorithm is applied to reach the best network plan satisfying our objectives. However, the authors do not address the economic aspects of radio network planning. In [23], a simple integer programming model for WCDMA radio network planning and optimization. Four heuristic algorithms, namely tabu search, simulated annealing, genetic algorithm and hill climbing local search, are used to find optimized network configurations. The experiments show that tabu search achieved the best performance and outperformed the other heuristics, while the worst performance appeared with greedy algorithm, which was expected. Evolutionary Simulated Annealing did slightly worse than tabu search, but genetic algorithm was strongly significantly worse, even with a delicate search.

This paper analyzes planning aspects of $3 \mathrm{G}$ radio network planning aspects, and provides some insights into efficient network planning issues Section II presents a $3 \mathrm{G}$ mobile network planning model. Section III formulates the $3 \mathrm{G}$ radio 
network planning problem as an optimization process. Section IV proposes the optimization solution structure and procedure. Section V demonstrates experimentally that the proposed planning model and the tabu search algorithm can efficiently find good solutions for realistic $3 \mathrm{G}$ planning scenarios. Section VI concludes the paper.

\section{3G RADIO Network PlanNING MODEL}

In this section, $3 \mathrm{G}$ radio downlink planning problem is used as an example to illustrate the planning process [13][14][15], which defines the decision variables, design constraints and objective functions.

\section{A. Working Area and Traffic}

A working area is the part of a geographical region where the mobile network needs to be deployed. Two basic discrete sets of points are identified on it.

A set of sites are candidates for the positioning base stations, denoted by $S=\{1, \ldots, m\}$, where a base station (BS) can be installed and that an installation and maintenance cost $C_{j}$ is associated with each candidate site $j, j \in S$. Each site is defined by its coordinates $(x, y)$, and eventually by $\mathrm{z}$ (height above sea level).

A set of traffic test points (TPs) $I=\{1, \ldots, n\}$ are described as in [13]. Each TP $i \in I$ can be considered as a centroid where a given amount of traffic $d_{i}$ is requested and where a certain level of service (measured in terms of SIR) must be guaranteed [11]. The required number of simultaneously active connections for TP $i$, denoted by $u_{i}$, turns out to be a function of the traffic, i.e., $u_{i}=\phi\left(d_{i}\right)$. The mobile terminals are located on TPs, where the network must overcome a signal quality threshold $S I R_{\text {min }}$, to ensure a given quality of service (QoS). The value of the threshold depends on the service type. To check the signal quality threshold on each TP, the field strength has to be computed on each point.

\section{B. Decision Variables}

In general, a BS antenna can be in one out of $q$ different configurations, denoted by set $L=\{1, \ldots, q\}$. A configuration represents a sextuplet $\mathrm{BS}=$ (location, type, height, tilt, azimuth, power). This accounts, for instance, for a variable tilt selected out of a set of possible angles with respect to the vertical axis, and for a variable height selected from a finite set of values with respect to the ground level. Since propagation gains depend on the BS antenna configuration, we denote by $g_{i j}^{w}$ the propagation gain from TP $i$ to potential site $j$ if the $\mathrm{BS}$ antenna is in configuration $w$. The decision variables are needed for each configuration:

$$
x_{i j w}= \begin{cases}1 & \text { if TP } i \text { assigned to } \mathrm{BS} j \\ & \text { with configuration } w \\ 0 & \text { otherwise }\end{cases}
$$

$y_{j w}= \begin{cases}1 & \text { if } \mathrm{BS} j \text { with configuration } w \\ 0 & \text { otherwise }\end{cases}$

for $i \in I, j \in S$ and $w \in L$. Once these basic decision variables have been determined, other dependent system variables, such as loading factors and SIR for each mobile terminal, etc., can be easily derived from.

\section{Design Constraints}

Constraints (3) make sure that each TP $i$ is assigned to at most one BS. Note that by restricting the assignment variables $x_{i j w}$ to take binary values, it is required that in every feasible solution all active connections must be assigned to a single BS.

$\sum_{j \in S} \sum_{w \in L} x_{i j w} \leq 1$

If the left terms of constraints (3) were forced to be equal to one, that is, each TP must connect to a BS, the results would too demanding for network resources. In some cases, a feasible solution would not be found. Therefore, constraints (3) are relaxed and intentionally allow some TPs not to be assigned.

Constraints (4) are called minimum service requirements [11], which ensure that service is available in the working area that have at least a proportion $\beta$ of all traffic demand nodes $(0<\beta<1)$. The parameter $\mu_{i}$ is the required number of simultaneously active connections for TP $i$.

$\sum_{j \in S} \sum_{w \in L} \sum_{i \in I} \mu_{i} x_{i j w} \geq \beta . \sum_{i \in I} \mu_{i}$

In our model, the cost of a BS involves the cost of site installation and cost of the configuration:

$C_{j}\left(y_{j w}\right)=C_{j}^{S}+C_{j}^{L}$

The overall cost of a radio access network in the predefined working area, $C(y)$, is the sum of the non-configuration cost of each BS antenna $C_{j}^{S}$, and its associated configuration cost $C_{j}^{L}$, i.e., $C(y)=\sum_{w \in L} \sum_{j \in S} y_{j w} . C_{j}\left(y_{j w}\right)$. The vector $\boldsymbol{y}$ is defined by constraints (2). Cost is an extremely important factor for choosing an adequate network configuration. Denote $C_{\max }$ an externally given ceiling cost, or a budgetary limit in total monetary investment. In most cases, it is practical to consider the budget constraints (6).

$\sum_{w \in L} \sum_{j \in S} y_{j w} \cdot C_{j}\left(y_{j w}\right) \leq C_{\max }$ 
among the coherence constraint $x_{i j w} \leq y_{j w}$, which ensures

$x_{i j w} \leq \min \left\{1, \frac{g_{i j}^{w} P_{\max }}{P_{t a r}}\right\} y_{j w}$

that $\mathrm{TP} i$ is only assigned to site $j$ if a $\mathrm{BS}$ with configuration $w$ is installed in $j$, and the power limit on a single connection from $\mathrm{BS} j$ to TP $i$ :

$$
\frac{P_{t a r}}{g_{i j}^{w}} x_{i j w} \leq P_{\max } y_{j w}
$$

where $P_{\max }$ is the maximum emission power for the connection from site $j$ to TP $i$ and $P_{t a r} / g_{i j}^{w}$ corresponds to the emission power required by $\mathrm{BS} j$ to guarantee the target received power Ptar at TP $i$. For each pair of site $j \in S$ and TP $i \in I$, constraints (8), which are active only if TP $i$ is assigned to $\mathrm{BS} j$ (i.e., $x_{i j w}=1$ ), correspond to the signal quality requirement. Finally, constraints (9) impose an upper limit $P_{\text {tot }}$ on the total emission power of every BS. Please note that the subscripts $i \in I, j \in S, w \in L$ are applicable to all the constraints in this paper.

$$
\sum_{i \in I} \frac{P_{t a r}}{g_{i j}^{w}} x_{i j w} \leq P_{t o t} y_{j w}
$$

In addition to the constraints (8) and (9), the quality of service constraints should be emphasized. Since in a powerbased power control mechanism $P_{t a r} / g_{i j}^{w}$ is the power that needs to be emitted from a BS with configuration $w$ in site $j$ to guarantee a received power of $P_{t a r}$ at TP $i$. For each connection between a BS installed in $j$ and a TP $i$ falling in a sector of this BS, the SIR constraints can be expressed as follows [13]:

$$
\frac{P_{\text {tar }} x_{i j w}}{I_{\text {int } e r}+I_{\text {int } r a}} \geq S I R_{\min } x_{i j w}
$$

Equation (11) represents the total interference incurred in the same cell, whereas Equation (12) describes the interference from all the other BSs, measured at mobile unit $i$ in the area.

$$
I_{\text {intra }}=\alpha\left(\sum_{\substack{k \in I_{j}^{\sigma_{i j w}} \\ x_{k j w}=1}} u_{k} \frac{g_{i j}^{w} P_{t a r}}{g_{k j}^{w}} x_{k j w}-P_{t a r}\right)
$$

$I_{\text {inter }}=\sum_{v \in L} \sum_{\substack{l \in S \\ l \neq j}} \sum_{\substack{k \in I_{l}^{\sigma_{l} l v} \\ x_{k l}=1}} u_{k} \frac{g_{i l}^{v} P_{t a r}}{g_{k l}^{v}} x_{k l v}$

where for any site $l \in S, w \in L$, the index set $I_{l}^{\sigma} i l w$ denotes the set of all TPs in $I$ that fall within the sector $\sigma_{i l w}$ of the BS with configuration $w$ and location $l$, which contains TP $i$. The parameter $(0<\alpha<1)$ is the wideband CDMA signal orthogonality factor. The parameter $\mu_{k}$ is the required number of simultaneously active connections for TP $k$.

For any single connection between a BS located at site $j$ and a TP $i$ falling in one of its sectors (denoted by $\sigma_{i j w}$ ), the numerator of the left-hand-side term in constraints (10) corresponds to the power of the relevant signal received at TP $i$ while the denominator amounts to the total interference due to all other active connections in the working area. Indeed, the first summation term expresses the total power received at TP $i$ that is directed from BS $j$ to every TP $k$ in $I_{j}^{\sigma_{k j w}}$, i.e., all TPs assigned to $\mathrm{BS} j$ with configuration $w$ and falling within the same sector as TP $i$, from which the received power $P_{t a r}$ of the relevant signal is subtracted. The second summation term expresses the interfering power received at TP $i$ that is directed from all BSs $l$, with $l \neq j$, to other TPs falling within the sectors $\sigma_{i l v}$ of each BS $l$ that do also contain TP $i$.

\section{Problem Formulation}

$3 \mathrm{G}$ planning problem can be formulated as an optimization problem. The available cell sites $(S)$, the traffic demand nodes $(I)$ with capacity requirements $(\mu)$, configuration set $(L)$ are fixed input parameters. Building upon the work by $[10][11][12][13]$, we propose three formulations of the $3 \mathrm{G}$ radio network planning problem. In all cases, the following variables are the basic decision variables. Based on these basic decision variables, most of radio access network parameters can be derived from [18][19].

1) The number of selected base stations and their configuration, denoted by multi-dimensional vector $y$.

2) The capacity assignment matrix, $\boldsymbol{x}$.

3) The power assignment vector (mobile transmitter participation), $\boldsymbol{p}$.

\section{Minimal Cost Planning}

A practical objective deals with the price of the solution in terms of installation and provision costs. In this formulation, the goal of the planning is to achieve as low cost as possible.

Minimize

$$
\sum_{w \in L} \sum_{j \in S} y_{j w} \cdot C_{j}\left(y_{j w}\right)
$$

Instead of optimizing the technical performance, such as coverage outage, which would potentially lead to a network with unnecessary high resource usage, we choose the objective for minimizing the network cost. The planning objective is defined by the formulation (13), which is subject 
to constraints (3)-(10).

\section{Maximum Capacity Planning}

A more real-life formulation of the $3 \mathrm{G}$ radio network planning problem is to aim for maximizing the satisfied capacity demands. The optimization problem can be written as:

Maximize $\sum_{w \in L} \sum_{j \in S} \sum_{i \in I} \mu_{i} x_{i j w}$

The objective function is the sum of the served capacity demands. If $x_{i j w}=1$, then $\mu_{i}$ at the location $i$ is served by BS $j$ with configuration $\mathrm{w}$. If $x_{i j w}=0$, then traffic $\mu_{i}$ at the location $i$ is in outage. The formulation (14) is subject to constraints (3)(10).

\section{Combined Cost and Capacity Planning}

This model explicitly considers the trade-off between the revenue potential of each $\mathrm{BS}$ site with its cost of installation and configuration. This trade-off is subject to $Q o S$ constraints in terms of sufficient SIR ratios (constraints (10)). The objective of the model is to maximize the total annual profit generated by the cellular network operator, which is equal to the total annual revenue minus the annual costs. Mathematically we have:

$$
\begin{gathered}
{\left[\theta \sum_{w \in L} \sum_{j \in S} \sum_{i \in I} \varepsilon \mu_{i} x_{i j w}{ }^{-}\right.} \\
\left.\sum_{w \in L} \sum_{j \in S} y_{j w} . C_{j}\left(y_{j w}\right)\right]
\end{gathered}
$$

$\varepsilon$ denotes the annual revenue (\$) generated by each channel utilized in the working area. $\theta$ is a weighting factor. Relation (15) represents the maximum profit optimization when $\theta=1$.

All three formulations (13), (14) and (15) are subject to constraints $(3)-(10)$.

\section{SOLUtion Procedure}

In order to apply tabu search to solve the planning problem, we need to define:

1) An initial feasible solution.

2) Representations for feasible solutions.

3) Neighborhoods for feasible solutions.

4) Search techniques for neighborhoods, and

5) Evaluation methods for gains in objective values.

\section{A. Solution Structure and Neighborhood}

Tabu search is a local search algorithm [24]. A local search algorithm starts from a candidate solution and then iteratively moves to a neighbor solution. This is only possible if a neighborhood relation is defined on the search space. Typically, every candidate solution has more than one neighbor solution; the choice of which one to move to is taken using only information about the solutions in the neighborhood of the current one, hence the name local search.

Decision variables are the BS locations, their powers, their antenna heights, etc. Given these decision variables, a radio access network configuration can be defined as a set of vectors $\left[\left(p_{l}, h_{l}, \ldots\right), \ldots,\left(p_{m}, h_{m}, \ldots\right)\right]$, which can be represented abstractly by the network configuration vector $y$. In practice, configuration parameters can take values from a certain range.

After vector $\boldsymbol{y}$ is decided, every traffic demand node TP $i \in I$ is assigned to a serving $\mathrm{BS}$ using a capacity assignment algorithm, described later in this section, that is, to determine the capacity assignment pattern, denoted by vector $\boldsymbol{x}$.

Each feasible search space point, denoted by $\boldsymbol{J}(\boldsymbol{x}, \boldsymbol{y})$, is a particular set of locations, powers, heights, and other configurations for each $\mathrm{BS}$, and a particular assignment pattern of traffic demand nodes to each selected BS satisfying the various constraints. To generate a new neighbor, two sets of neighborhood generating operators are required, one that moves the locations and configurations of BSs and another that changes the capacity assignment pattern for each BS. The first set of operators is defined as follows:

1) On-Off: a BS site is chosen randomly. If there is a BS at the site, it is removed. If there is no BS at the site yet, a new $\mathrm{BS}$ is placed at the site.

2) Local Move: one of the decision variables (power, height, or other configuration parameters) of a randomly chosen BS is appointed randomly, and the new neighbor is generated by taking its value one size above or one size below its current value.

\section{B. Capacity Assignment Algorithm}

The second set of operators is the capacity assignment algorithm. Given the locations of BSs, their powers, heights and other configurations, demand nodes I should be assigned first to the available BS that has the largest signal attenuation factor before establishing connections to other BSs [13]. The algorithm works like this:

1) Step 0: Start with a given radio access network configuration $\boldsymbol{y}$.

2) Step 1: For each $i \in I$, calculate minimum power $P_{t a r} / g_{i j}^{w}$ according to propagation matrix $G=\left[g_{i j}^{w}\right]$; assign demand node $i$ to its closest $\mathrm{BS} j$, requiring the minimal transmit power; calculate $\boldsymbol{x}$. In this step, constraints (7) are automatically satisfied.

3) Step 2: If $\boldsymbol{x}$ from Step 1 and $\boldsymbol{y}$ satisfy constraints (9), go to Step 3; otherwise repeat the process: randomly select and disconnect a demand node $i$ belonging to the overcrowded BS $\mathrm{j}$, which will reduce its transmit power accordingly, until constraints (9) are satisfied.

4) Step 3: If $\boldsymbol{x}$ from Step 2 and y satisfy constraints (10), go to Step 4; randomly select and disconnect a demand node $\mathrm{i}$ belonging to the overcrowded $\mathrm{BS} \mathrm{j}$, which will reduce its transmit power accordingly, until constraints (10) are satisfied. 
5) Step 4: Output final capacity assignment vector $\boldsymbol{x}$.

\section{Initial Solution Generation}

An initial network configuration is generated by intuitively (randomly) placing BSs at potential locations with a uniform distribution, in such a way that the number of initial locations is approximately half the total number of possible locations in our implementation, and assigning their powers, antenna heights, and other configuration parameters within the respective feasible ranges. Then every traffic demand node TP $i$ is assigned to a closest serving BS using the demand assignment algorithm, described earlier in this section.

The next step is the optimization process. The heuristic algorithm receives as input the initial network configuration, finds better configurations by improving the measure of performance in successive iterations and returns a final good quality network configuration and traffic assignment pattern.

\section{Tabu Search}

Tabu search is a mathematical optimization method, belonging to the class of local search techniques. Tabu search enhances the performance of a local search method by using memory structures: once a potential solution has been determined, it is marked as "taboo" ("tabu" being a different spelling of the same word) so that the algorithm does not visit that possibility repeatedly. Tabu search is attributed to [16].

The tabu search implementation for the cell planning and optimization problem may be described as follows [13]:

1) Step 0: $l \leftarrow 0$. The counter of the algorithm iterations, $l$, is initialized. An initial solution $\boldsymbol{J}^{\mathrm{o}}(\boldsymbol{x}, \boldsymbol{y})$ is chosen randomly in such a way that the number of BSs is equal to half the total potential locations, and that the power, the height and other configuration parameters of a selected BS are chosen randomly from an element of the corresponding feasible set. Then, the capacity assignment algorithm is applied to generate the capacity assignment pattern. Calculate the objective function, $f\left(\boldsymbol{J}^{\boldsymbol{o}}\right)$ from relation (13), (14) or (15). The set of taboo and aspirant moves is initialized, i.e., $T(l)=\varnothing$, and $A(l)$ $=\varnothing$. A candidate list $M(l)$ is initialized.

2) Step 1: If the stop criterion is satisfied, the procedure ends and a transition to Step 9 is performed.

3) Step 2: The set of move operators $M$ is applied to solution $J^{l}$, and hence, a new set of candidate solution list $M(l)$ is produced. Each move produces a new neighbor of the current search space point.

4) Step 3: The $M(l)$ set is examined to satisfy the constraints (8), (9), and (10) forms the new set of solutions $N(l)$ that are neighbouring to solution $J^{l}$.

5) Step 4: The set of solution, $C(l)$, that are candidate for obtaining the best solution status, and therefore, for replacing solution $J^{l}$ in the next algorithm iteration, is formed through the relation $C(l)=N(l)-T(l)$. Moreover, solution $J^{l}$ is appended in the $C(l)$ set.

6) Step 5: The $A(l)$ set is formed. More specifically, the objective function value that is scored by the solutions in the $T(l)$ set is computed. Those solutions that improve the objective function by more than a given level are removed from the $T(l)$ set and placed in the $A(l)$ set.

7) Step 6: The set of solutions $C(l)$ is enhanced through the relation $C(l)=C(l) \cup A(l)$.

8) Step 7: The solution of the $C(l)$ set that is the best in improving the objective function becomes the best solution that will be used in the next algorithm iteration.

9) Step 8: $l \leftarrow l+1$. The next algorithm iteration is prepared. Therefore, the set of tabu moves that will be used in the next algorithm iteration is updated through the relation $T(l$ $+1)=T(l) \cup M(l)$. Moreover, solutions that have stayed in the taboo set for more than a given number of algorithm iterations are removed form the $T(l+1)$ set. Finally, $A(l+1)=$ $\varnothing$ and $C(l)=\varnothing$. A transition to Step 1 is performed.

10) Step 9: End.

An experimental analysis of the performance of four search algorithms for the problem of designing the optimal location of base stations in a mobile communication system [21][23] Random Walk, Simulated Annealing, Tabu Search and Genetic Algorithms. It is found that Genetic Algorithms and Tabu Search both perform well with Tabu Search providing the most consistent final cost value on multiple runs. So, it is reasonable to use Tabu Search in this paper.

\section{EXPERIMENTAL RESULTS}

Numerical experiments are made for $3 \mathrm{G}$ downlink radio network planning. The system performance evaluations are based on 3G WCDMA system specification. The optimization strategies used in these experiments are also applicable to other systems (e.g., TD-SCDMA, CDMA 2000). We will present in detail a realistic planning scenario in this section.

\section{A. Impact of Planning Objectives}

In pure capacity maximization planning, the network cost is not included in the optimization process, which may result in network configurations with unnecessarily high cost. In pure cost minimization planning, the capacity is not included in the optimization process, which may result in network configurations with unacceptable low capacity. Our purpose with the following quantitative examples is to illustrate the tradeoff between capacity and cost. Table 1 contains important planning input data.

TABLE I

3G CELl PLANNING DATA

\begin{tabular}{|l|c|}
\hline \multicolumn{1}{|c|}{ Parameters } & Values \\
\hline Mobile antenna height & $1.8 \mathrm{~m}$ \\
\hline Frequency & $2 \mathrm{GHz}$ \\
\hline Mobile antenna gain & $0 \mathrm{db}$ \\
\hline BS antenna gain & $14 \mathrm{db}$ \\
\hline$S I R_{\min }$ & 0.009789 \\
\hline$E_{b} / I_{o}$ & $7 \mathrm{db}$ \\
\hline Processing gain & 512 \\
\hline Mobile receiver sensitivity & $-110 \mathrm{dBm}$ \\
\hline WCDMA orthogonality & 0.7 \\
\hline Thermal noise density & $-130 \mathrm{dBm} / \mathrm{Hz}$ \\
\hline Annual revenue per channel & $\$ 10,000$ \\
\hline
\end{tabular}


Let us assume that the task is to design $3 \mathrm{G}$ downlink radio access network to provide conversational service over a predefined urban working area $1.5 \mathrm{~km} \times 1.5 \mathrm{~km}$. There are 500 demand nodes (n) uniformly distributed, and for the sake of simplicity, each demand node channel requirement $\mu_{i}$ takes one value from the set $\{1,2,3\}$ for each $i \in I$. There are 100 potential candidate BS locations $(m)$ which are randomly generated with an uniformly distribution. Assume that annual site non-configuration cost is $\$ 100 \mathrm{~K}$ for each potential site. BS antennas are assumed to be omnidirectional at every site. For the sake of presentation clarity, we define the feasible power- and antenna height sets for all sites uniformly. The three power and height values, as well as their associated configuration costs, are shown in Table 2. For simplicity, we assume the power-based power control mechanism is adopted, because it requires much less computational effect. COST 231 Hata urban propagation model [17] is used to generate configuration gain matrix $\mathrm{G}=\left[g_{i j}^{w}\right]$.

TABLE II

Feasible VAlues for ANTENNA POWER, Height AND Costs

\begin{tabular}{|c|c|c|c|}
\hline \multicolumn{2}{|c|}{ Max transmit power } & \multicolumn{2}{|c|}{ BS antenna height } \\
\hline $\begin{array}{c}\text { Permitted } \\
\text { values } \\
\text { (watts) }\end{array}$ & $\begin{array}{c}\text { Cost } \\
\text { (unit \$K) }\end{array}$ & $\begin{array}{c}\text { Permitted } \\
\text { values } \\
\text { (m) }\end{array}$ & $\begin{array}{c}\text { Cost } \\
\text { (unit \$K) }\end{array}$ \\
\hline 20 & 100 & 10 & 10 \\
\hline 40 & 150 & 20 & 20 \\
\hline 80 & 200 & 30 & 30 \\
\hline
\end{tabular}

From Table 2, intuitively, BS antenna height configuration costs are not significant when compared to other cost components. This means that the antenna heights are not important in cost optimization, but important in capacity optimization, because antenna heights are a dominating factor in determining coverage area and received power strengths at traffic demand node points. We apply tabu search to solve the above planning problem, but using alternative objective functions, according to the following three scenarios:

1) Capacity Planning (CAP): Cost is completely disregarded during the optimization, the objective function is formulated in relation (14) to maximize the served traffic in the working area (or minimize the number of unserved traffic points where some constraints are not satisfied (outage). For simplicity, we call this scenario the pure capacity planning.

2) Cost Planning (COST): Capacity factor is completely ignored during the optimization, the objective function is formulated in relation (13) to minimize the total cost and hopefully find the cheapest feasible network configuration during the optimization process.

3) Combined Cost and Capacity Planning (COM): Cost is part of the objective function, according to relation (15). The weighing factor $\theta$ allows us to give priority to either minimizing cost or maximizing capacity. To find an appropriate value for $\theta$, a number of alternative values have been applied, and the results are subject to comparisons based on a large number of independent tabu search executions. Fig. 1 summaries the results of four representative values, $\theta=0.1$, 1.0 and 10 .

Low $\theta$ value results in low success in terms of finding high capacity feasible network configurations, which can be attributed to the possibility to sacrifice one or more traffic demand nodes to obtain a cheaper network configuration. This phenomenon is demonstrated by the low traffic service ratio (capacity) as well as by the apparently low total cost figures, as it is exemplified by the $\theta=0.1$ case in Fig. 1. The $\theta=1$ case already represents a situation where network configurations have on average a higher capacity and cost than feasible configurations with low network cost. The $\theta=$ 10 case makes solutions cluster than $\theta=1$. Experiment show that Increasing $\theta$ further does not lead to additional capacity improvement.
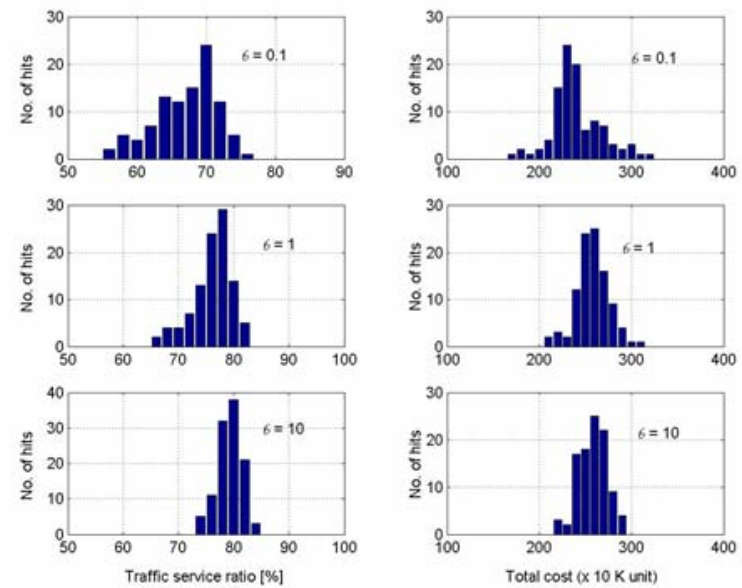

Fig. 1. Resulting overall traffic coverage ratios and total cost histograms for three different capacity weighting factors, $\theta=0.1,1$ and 10 .

Fig. 2 summaries the results of the three planning cases CAP, COST, and COM in terms of the total obtained network configuration costs. The histograms are generated from the results of 100 independent tabu search runs for each of the three cases. We use the same parameters and test cases as above for $C A P, C O S T$, and $C O M$, and apply $\theta=1$ for $C O M$. It can be seen that pure cost optimization case yields considerable cost reduction when at least $40 \%$ traffic demand must be satisfied. It is believed that pure cost optimization case would not select any BS if we did not specify a minimum traffic satisfaction requirement. 

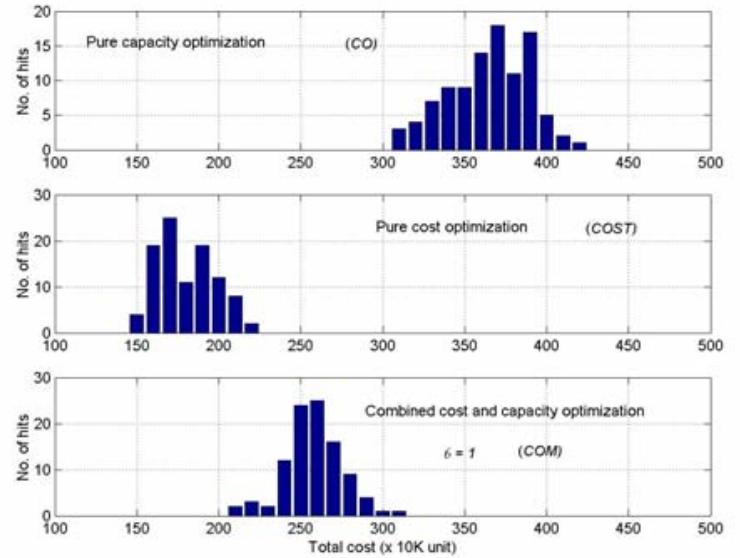

Fig. 2. Resulting total cost values for pure capacity optimization, pure cost optimization, combined cost and capacity optimization $(\theta=1)$.

Likewise, pure capacity planning yields considerable cost increase. Here we assume that the budgetary constraint allows no more than 20 BS sites installed. The combined cost and capacity optimization case lies somewhere between two extreme cases, striking a meaningful tradeoff. For explanation we can look at Table 3, which contains that statistical results of 100 resulted network configurations found by tabu search for each objective (CAP, COST, COM).

TABLE III

SUMMARY OF ALLOCATED RESOURCES FOR SCENARIOS CAP, COST, COM

\begin{tabular}{|c|c|c|c|c|c|c|}
\hline \multirow{2}{*}{ 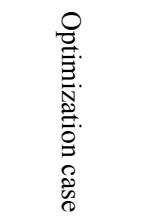 } & \multicolumn{2}{|c|}{$\hat{\theta}$} & \multicolumn{2}{|c|}{ ठ } & \multicolumn{2}{|c|}{$\begin{array}{l}\approx \Omega \\
\| \\
=\end{array}$} \\
\hline & 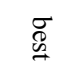 & 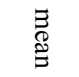 & $\begin{array}{l}\sigma_{0} \\
\stackrel{n}{\sim}\end{array}$ & $\begin{array}{l}\text { E्ठ } \\
\stackrel{\mathscr{E}}{E}\end{array}$ & $\begin{array}{l}\mathscr{\sigma} \\
\stackrel{\varnothing}{\sim}\end{array}$ & 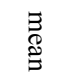 \\
\hline No. of BS & 12 & 14.78 & 5 & 6.60 & 8 & 10.71 \\
\hline Total power & 56.81 & 57.29 & 53.98 & 55.46 & 55.05 & 56.17 \\
\hline $\begin{array}{l}\text { Average } \\
\text { power }\end{array}$ & 46.02 & 45.59 & 46.99 & 47.27 & 46.23 & 45.87 \\
\hline $\begin{array}{c}\text { Average } \\
\text { height }\end{array}$ & 17.05 & 16.08 & 22.00 & 22.12 & 17.50 & 17.48 \\
\hline Total cost & 3060 & 3745 & 1410 & 1866 & 2040 & 2727 \\
\hline
\end{tabular}

Measurement Unit: power $[\mathrm{dBm}]$, height $[\mathrm{m}]$ and $\operatorname{cost}[\$ \mathrm{~K}]$

The resource utilization of the networks is measured by the average number of BS transmitters in the networks, average total power, average height of antennas in the networks and the average cost. Besides the average values over the 100 runs, we indicate the parameters of the best found network in terms of cost.

Comparing the statistical values, the total power usage of these three cases are very similar. In pure capacity planning, the results favor networks with a larger number of lower BSs. In contrast to that, networks of the pure cost planning contain fewer BSs with higher structural antenna.

Comparing the best achievable results of $C A P$ and $C O M$, the total power usage (i.e., CAP, $56.81 \mathrm{dBm}$ and $C O M, 55.05$
$\mathrm{dBm})$ and the average antenna height $(C A P, 17.05 \mathrm{~m}$ and $C O M, 17.50 \mathrm{~m})$ are approximately the same for both objectives, but the number of BSs is decreased from 12 (CAP) to $8(C O M)$. This result indicates that the network remains "over-provisioned" under $C A P$, that is, the planning does not attempt to remove unnecessary BSs, neither to cut back excessive power.

\section{B. Impact of Cost Configurations}

In this section, we present the effect of different cost configurations in the same planning problem. The trials are performed in a $3 \mathrm{G}$ downlink radio network planning task. Assume that power-based power control mechanism is adopted. In this case, the urban area is $1.5 \mathrm{~km} \times 1.5 \mathrm{~km}$. There are 500 demand nodes $(n)$ uniformly distributed, and for the purpose of simplicity, each demand node channel requirement $\mu_{i}$ take one value from the set $\{1,2,3\}$ for each $i \in I$. There are 100 potential candidate BS locations $(m)$ which are randomly generated with an uniformly distribution. Assume that annual site non-configuration cost is $\$ 100 \mathrm{k}$ for each potential site. BS antennas are assumed to be omnidirectional at every site. For simplicity, we assume that all BS antennas have the same height $(10 \mathrm{~m})$, because in $3 \mathrm{G}$ radio network planning the BS antenna height cost is negligible compared to other cost components, e.g., BS equipment cost (power related). The feasible set of power values is $\boldsymbol{P}=\{20,40,80$, $120\}$ [w]. Two different cost configurations are applied during the planning process as defined by Table 4 (The cost figures are fictitious and for illustration purpose only). The objective function of this planning task is $\mathrm{COM}$ with $\theta=1$.

TABLE IV

TWO SETS OF BS COST CONFIGURATIONS

\begin{tabular}{|c|c|c|}
\hline $\begin{array}{c}\text { Max power } \\
\text { (watts) }\end{array}$ & $\begin{array}{c}\text { Cost } \\
\text { configuration } 1 \\
\text { (x \$K) }\end{array}$ & $\begin{array}{c}\text { Cost } \\
\text { configuration 2 } \\
\text { (x \$K) }\end{array}$ \\
\hline 20 & 100 & 100 \\
\hline 40 & 250 & 150 \\
\hline 80 & 400 & 200 \\
\hline 120 & 500 & 250 \\
\hline
\end{tabular}

For both cost configurations, the optimization of the planning process is performed by tabu search algorithm, 100 runs. The resulting network configurations are evaluated by the frequency of the BS antennas with power values of $\boldsymbol{P}$. The statistics presented in Fig. 3 follow the cost configurations. In the cost configuration 1 , the cost of antenna power drastically increases for larger values and the site cost relatively low. According to the expectations, the resulting BS antenna assignment in Fig. 3 (left subfigure) is featured with a large number of BS antennas, on average 15.83, with low power. 

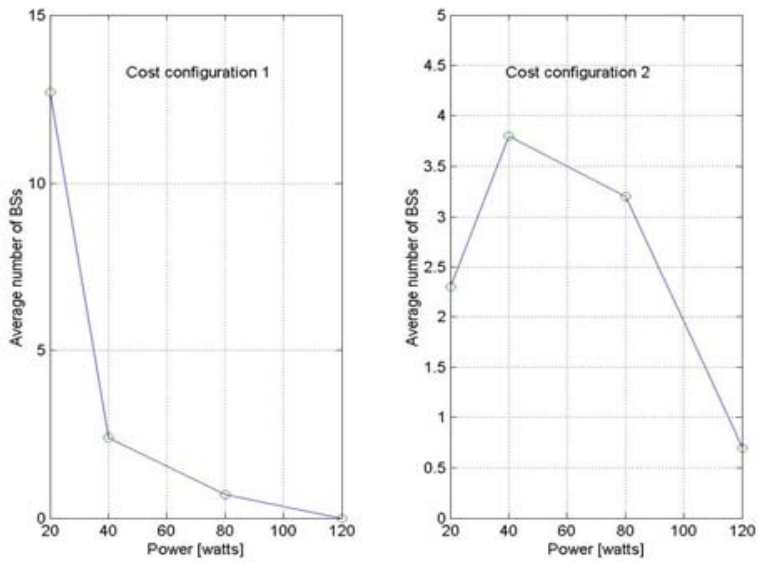

Fig. 3. For cost 1, the average BS number is 15.83 for cost configuration (left subfigure). For cost 2, the average BS number is 10.15 (right subfigure).

In the cost configuration 2, we repeat the optimization with a modified cost configuration where the BS antenna power is relatively cheap. As the results show in Fig. 3 (right subfigure), the optimization reacts with different solution to the input. The average number of BS antennas is 10.15. Due to the cost configuration and its relation to the size of the coverage area per BS, the middle size BS antennas are preferred. The use of large BS antennas requires also some small gap filling BS antennas. Due to the high cost for $\boldsymbol{P}=80$, $120 \mathrm{w}$, the number of BS antennas with these power values is low.

\section{Performance Evaluation of Tabu Search}

In order to evaluate the performance of Tabu search, we compare it with greedy algorithm and simulated annealing algorithm experimentally [20]. These three optimization algorithms are tested over the same parameters as listed in Tables 1 and 2, and the same planning case $\operatorname{COM}(\theta=1)$. We focus on evaluation of the quality of the solutions in terms of objective function value, which in our test case represents the total profit. Three algorithms always find feasible solutions. In each of 30 test runs, Tabu search consistently yields better optimization results in the objective function value (total profit) in comparison with simulated annealing and greedy algorithm, with average improvement rate $0.85 \%$ over simulated annealing and $2.13 \%$ over greedy algorithm respectively. In terms of execution time, greedy algorithm is the fastest among the three, followed by simulated annealing and Tabu search. Here, the execution time is not as important as the improvement in optimization results, because for largesize cellular network projects (in the order of tens of millions of dollars), a small improvement in optimization results can translate it into less capital investment at the beginning of the project and a good cost saving in the long run; for example, for a mobile operator whose network building cost is $500 \mathrm{M}$ per year, the $0.85 \%$ and $2.13 \%$ improvements are equivalent to $4.25 \mathrm{M}$ and $10.65 \mathrm{M}$ annual investment savings respectively, which can make a difference.

\section{CONCLUSION}

This paper mainly focuses on the economic aspects of mobile network planning. Firstly, instead of just minimizing the cost as most previous works do, we consider alternative objectives, which better reflect the dynamics of the various requirements of potential mobile operators. Secondly, the decision variables and traffic capacity can be embedded in the objective function and are optimized in a single integrated step. This can result in the tradeoff between the cost reduction and the capacity improvement, compared with approaches where they are considered separately. Finally, because it deals with the static simulations of $3 \mathrm{G}$ networks, the results from this paper provide the low bound for the network infrastructure investment. The dynamic aspects, such as soft handoff, are not necessarily considered. Future work should focus on developing better algorithms and models which incorporate multi-traffic scenarios, existing network expansion, and time.

\section{REFERENCES}

[1] M. R. Garey and D. S. Johnson. Computers and Intractability: a guide to the theory of NP-completeness, W. H. Freeman and Company, 1979.

[2] H. R. Anderson and J. P. McGeehan, "Optimizing microcell base station locations using simulated annealing techniques," Proceedings of IEEE Vehi. Tech. Conf., vol. 2, June 1994, pp. 858-862.

[3] H. D. Sherali, C. M. Pendyala, and T. S. Rappaport, "Optimal location of transmitters for microcellular radio communication system design," IEEE J. Selected Areas in Communications, vol. 14, No. 4, May 1996, pp. 662-673.

[4] D. Stamatelos and A. Ephremides, "Spectral efficiency and optimal base station placement for indoor wireless networks, " IEEE J. Selected Areas in Communications, vol. 14, No. 4, May 1996, pp. 651-661.

[5] K. W. Cheung, and R. D. Murch, "Optimising indoor base station locations in coverage and interference limited indoor environments," Proceedings of IEE Communications, Dec. 1998, pp. 445-450.

[6] R. K. Rawnsley, and S. Hurley, "Towards automatic cell planning," Proceedings of the 11th IEEE International Symposium on Personal, Indoor, and Mobile Radio Communications, Sept. 2000, pp. 1583-1588.

[7] J. Kalvenes, J. Kennington, and E. Olinick, "Hierarchical cellular network design with channel allocation," Technical Report 01-EMIS-08, Southern Methodist University, 2001.

[8] A. Dutta and V. Hsu, "Cellular network design site selection and frequency planning," Annals of Operations Research, vol. 106, 2001, pp. 287-306.

[9] C. Y. Samuel, CDMA RF System Engineering, Artech House, 1998.

[10] J. Laiho, A.Wacker, and T.Novosad (ed.), Radio Network Planning and Optimization for UMTS, John Wiley \& Sons Ltd. 2001.

[11] J. Kalvenes, J. Kennington, and E. Olinick, "Base station location and service assignment in WCDMA networks," Technical Report 02-EMIS03, Southern Methodist University, 2002.

[12] A. Ligeti and J. Zander, "Minimal cost coverage planning for single frequency networks," IEEE Transactions on Broadcasting, vol. 45, No.1, March 1999, pp. 78-87.

[13] E. Amaldi, A. Capone, and F. Malucelli, "Discrete models and algorithms for the capacitated location problem arising in UMTS network planning," Proceedings of ACM Dial-M, 2001, pp. 1-8.

[14] K. Tutschku and P. Tran-Gia, "Spatial traffic estimation and characterization for mobile communication network design," IEEE J. Selected Areas in Communications, vol. 16, No. 5, 1998, pp. 804-811.

[15] E. Amaldi, A. Capone, and F. Malucelli, "Base station configuration and location problems in UMTS networks," Proceedings of the 9th Conference on Telecommunication Systems, Modeling and Analysis, 2001.

[16] F. Glover, E. Taillard and D. de Werra, "A user's guide to tabu search," Annals of Operations Research, vol. 41, 1993, pp. 3-28. 
[17] COST 231 Final Report, Digital Mobile Radio: COST 231 View on the Evolution Towards 3rd Generation System, 1999.

[18] A. Eisenblatter, et al., "Modelling feasible network configurations for UMTS," ZIB-Report 02-16, March 2002.

[19] E. Melachrinoudis, and B. Rosyidi, "Optimizing the design of a CDMA cellular system configuration with multiple criteria," Annals of Operations Research, Vol. 106, 2001, pp. 307- 329.

[20] S. Kirkpatrick, C.D. Gelatt Jr., M.P. Vecchi, "Optimization by simulated annealing," Science 220, pp. 671-680, 1983.

[21] B. Krishnamachari, "Global optimization in the design of mobile communication systems," Master's thesis, Electrical Engineering, Cornell University 1999

[22] E.M. Elkamchouchi, M.E. Hassan, and M.A. Makar, "Cellular radio network planning using particle swarm optimization," 24th National Radio Science Conference, Egypt, 2007.

[23] J. Zhang, J.Yang, M. Aydin, and J.Y. Wu, "Mathematical modelling and comparisons of four heuristic optimization algorithms for WCDMA radio network planning," 8th International Conference on Transparent Optical Networks, Nottingham, U.K., 2006.

[24] E. Aarts and J.K. Lenstra, Local Search in Combinational Optimization, Princeton University Press, 2003.

[25] H. Holma and A. Toskala, WCDMA for UMTS, John Wiley \& Sons, 2002

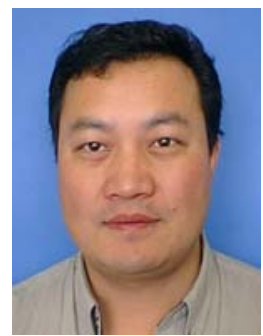

Yufei Wu received the B.Sc. and M.Sc., both in radio communications, from Nanjing University, Nanjing, China. He also received an MBA degree in 1995 from McGill University, and Ph.D. in mobile communications engineering from the University of Montreal (Ecole Polytechnique) in 2004, both from Montreal, Canada.

Since 2006, Dr. Wu has been an assistant professor at the University of Trinidad and Tobago. His research interests focus on mobile network planning and optimization, communication system simulations, mobile gaming and animation, and wireless anti-crime and security technology.

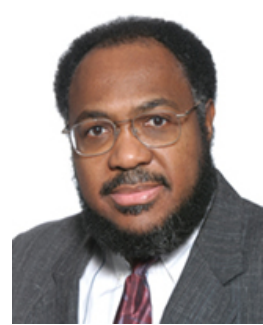

Samuel Pierre received the B.Eng. degree in civil engineering in 1981 from École Polytechnique de Montréal, Québec, the B.Sc. and M.Sc. degrees in mathematics and computer science in 1984 and 1985, respectively, from the UQAM, Montréal, the M.Sc. degree in economics in 1987 from the Université de Montréal, and the Ph.D. degree in Electrical Engineering in 1991 from École Polytechnique de Montréal. From 1987 to 1998, he was a Professor at the Université du Québec à TroisRivières prior to joining the Télé-Université of Québec, an Adjunct Professor at Université Laval, Ste-Foy, Québec, an Invited Professor at the Swiss Federal Institute of Technology, Lausanne, Switzerland, then the Universite Paris 7, France. He is currently a Professor of Computer Engineering at École Polytechnique de Montréal where he is Director of the Mobile Computing and Networking Research Laboratory (LARIM), chairholder of the NSERC ERICSSON Chair in Next Generations Mobile Networking Systems and Director of Mobile Computing and Netwking Research Group (GRIM). His research interests include wireline and wireless networks, mobile computing, artificial intelligence, and telelearning. He is a senior member of IEEE and a member of ACM. He is a Regional Editor of the Journal of Computer Science, an Associate Editor of IEEE Communications Letters, IEEE Canadian Journal of Electrical and Computer Engineering and IEEE Canadian Review, and serves on the editorial board of Telematics and Informatics edited by Elsevier Science. He has received many distinctions, such as the Prix Poly 1873 for excellence in teaching (2001 and 2005), Fellow of Engineering Institute of Canada (2003), among others. He is a Fellow of the Canadian Academy of Engineering (2008). 